\title{
Search for new physics in rare B decays
}

\section{OPAL Collaboration}

G. Abbiendi ${ }^{\mathrm{b}}$, K. Ackerstaff ${ }^{\mathrm{h}}$, P.F. Akesson ${ }^{\mathrm{c}}$, G. Alexander ${ }^{\mathrm{v}}$, J. Allison ${ }^{\mathrm{p}}$, K.J. Anderson i, S. Arcelli ${ }^{q}$, S. Asai ${ }^{w}$, S.F. Ashby ${ }^{a}$, D. Axen ${ }^{\text {aa }}$, G. Azuelos ${ }^{\mathrm{r}, 1}$, I. Bailey ${ }^{\mathrm{z}}$, A.H. Ball ${ }^{\mathrm{h}}$, E. Barberio ${ }^{\mathrm{h}}$, R.J. Barlow ${ }^{\mathrm{p}}$, J.R. Batley ${ }^{\mathrm{e}}$, S. Baumann ${ }^{\mathrm{c}}$,

T. Behnke ${ }^{\mathrm{y}}$, K.W. Bell ${ }^{\mathrm{t}}$, G. Bella ${ }^{\mathrm{v}}$, A. Bellerive ${ }^{\mathrm{i}}$, S. Bentvelsen ${ }^{\mathrm{h}}$, S. Bethke ${ }^{\mathrm{n}, 2}$, O. Biebel ${ }^{n, 2}$, A. Biguzzi ${ }^{\text {e }}$, I.J. Bloodworth ${ }^{a}$, P. Bock ${ }^{k}, J_{\text {. Böhme }}{ }^{n, 3}$, O. Boeriu ${ }^{j}$, D. Bonacorsi ${ }^{\text {b }}$, M. Boutemeur ${ }^{\text {ae }}$, S. Braibant ${ }^{\text {h }}$, P. Bright-Thomas ${ }^{\text {a }}$, L. Brigliadori ${ }^{\mathrm{b}}$, R.M. Brown ${ }^{\text {t }}$, H.J. Burckhart h, J. Cammin ${ }^{\text {c }}$, P. Capiluppi ${ }^{\text {b }}$, R.K. Carnegie ${ }^{\text {f }}$, A.A. Carter ${ }^{\mathrm{m}}$, J.R. Carter ${ }^{\mathrm{e}}$, C.Y. Chang ${ }^{\mathrm{q}}$, D.G. Charlton ${ }^{\mathrm{a}, 4}$, D. Chrisman ${ }^{\mathrm{d}}$, C. Ciocca ${ }^{\text {b }, \text { P.E.L. Clarke }}{ }^{\circ}$, E. Clay ${ }^{\circ}$, I. Cohen ${ }^{\mathrm{v}}$, O.C. Cooke ${ }^{\mathrm{h}}, \mathrm{J}_{\text {. Couchman }}{ }^{\mathrm{o}}$, C. Couyoumtzelis ${ }^{\mathrm{m}}$, R.L. Coxe ${ }^{\mathrm{i}}$, M. Cuffiani ${ }^{\mathrm{b}}$, S. Dado ${ }^{\mathrm{u}}$, G.M. Dallavalle ${ }^{\mathrm{b}}$, S. Dallison ${ }^{\mathrm{p}}$, R. Davis ${ }^{\mathrm{ab}}$, A. de Roeck ${ }^{\mathrm{h}}$, P. Dervan ${ }^{\mathrm{o}}$, K. Desch ${ }^{\mathrm{y}}$, B. Dienes ${ }^{\mathrm{ad}, 3}$, M.S. Dixit ${ }^{\mathrm{g}}$, M. Donkers ${ }^{\mathrm{f}}$, J. Dubbert ${ }^{\mathrm{ae}}$, E. Duchovni ${ }^{\mathrm{x}}$, G. Duckeck ${ }^{\text {ae }}$, I.P. Duerdoth ${ }^{\text {p }}$, P.G. Estabrooks ${ }^{\text {f }}$, E. Etzion ${ }^{\text {v }}$, F. Fabbri ${ }^{\text {b }}$, A. Fanfani ${ }^{\text {b }}$, M. Fanti ${ }^{\text {b }}$, A.A. Faust ${ }^{\text {ab }}$, L. Feld ${ }^{j}$, P. Ferrari ${ }^{1}$, F. Fiedler ${ }^{y}$, M. Fierro ${ }^{b}$, I. Fleck ${ }^{j}$, A. Frey ${ }^{\text {h, }}$ A. Fürtjes ${ }^{\text {h }}$, D.I. Futyan ${ }^{\text {p }}$, P. Gagnon ${ }^{\text {, J.W. Gary }}{ }^{\mathrm{d}}$, G. Gaycken ${ }^{\mathrm{y}}$, C. Geich-Gimbel ${ }^{\text {c }}$, G. Giacomelli ${ }^{\text {b }}$, P. Giacomelli ${ }^{\text {b }}$, D.M. Gingrich ${ }^{\text {ab, }, 1}$, D. Glenzinski i , J. Goldberg " , W. Gorn ${ }^{\mathrm{d}}$, C. Grandi ${ }^{\mathrm{b}}$, K. Graham ${ }^{\mathrm{z}}$, E. Gross ${ }^{\mathrm{x}}$, J. Grunhaus " , M. Gruwé ${ }^{\text {y }}$, P.O. Günther ${ }^{\text {c }}$, C. Hajdu ${ }^{\text {ac }}$, G.G. Hanson ${ }^{1}$, M. Hansroul ${ }^{\text {h}}$, M. Hapke ${ }^{\mathrm{m}}$, K. Harder ${ }^{\mathrm{y}}$, A. Harel " , C.K. Hargrove ${ }^{\mathrm{g}}$, M. Harin-Dirac ${ }^{\text {d }}$, A. Hauke ${ }^{\text {c }}$, M. Hauschild ${ }^{\text {h }}$, C.M. Hawkes ${ }^{a}$, R. Hawkings ${ }^{y}$, R.J. Hemingway ${ }^{\mathrm{f}}$, C. Hensel ${ }^{\mathrm{y}}$, G. Herten ${ }^{\mathrm{j}}$, R.D. Heuer ${ }^{\mathrm{y}}$, M.D. Hildreth ${ }^{\mathrm{h}}$, J.C. Hill ${ }^{\text {e }}$, P.R. Hobson ${ }^{\mathrm{y}}$, A. Hocker ${ }^{\mathrm{i}}$, K. Hoffman ${ }^{\mathrm{h}}$, R.J. Homer ${ }^{\mathrm{a}}$, A.K. Honma ${ }^{\text {h }}$, D. Horváth ac,5, K.R. Hossain ${ }^{\text {ab }}$, R. Howard ${ }^{\text {aa }}$, P. Hüntemeyer ${ }^{y}$, P. Igo-Kemenes ${ }^{\text {k }}$, D.C. Imrie ${ }^{y}$, K. Ishii ${ }^{\text {w }}$, F.R. Jacob ${ }^{\mathrm{t}}$, A. Jawahery ${ }^{\mathrm{q}}$, H. Jeremie ${ }^{\mathrm{r}}$, M. Jimack ${ }^{\text {a }}$, C.R. Jones e , P. Jovanovic ${ }^{\text {a }}$, T.R. Junk ${ }^{\text {f }}$, N. Kanaya ${ }^{\text {w }}$, J. Kanzaki ${ }^{\text {w, }}$ G. Karapetian ${ }^{\text {r }}$, D. Karlen ${ }^{\text {f }}$, V. Kartvelishvili ${ }^{\mathrm{p}}$, K. Kawagoe ${ }^{\text {w }}$, T. Kawamoto ${ }^{\text {w }}$, P.I. Kayal ${ }^{\text {ab }}$, R.K. Keeler ${ }^{\text {z }}$, R.G. Kellogg ${ }^{\mathrm{q}}$, B.W. Kennedy ${ }^{\mathrm{t}}$, D.H. Kim ${ }^{\mathrm{s}}$, K. Klein ${ }^{\mathrm{k}}$, A. Klier ${ }^{\mathrm{x}}$, T. Kobayashi ${ }^{\text {w }}$, M. Kobel ${ }^{\text {c }}$, T.P. Kokott ${ }^{\text {c }}$, M. Kolrep ${ }^{\mathrm{j}}$, 
S. Komamiya ${ }^{\text {w }}$, R.V. Kowalewski ${ }^{\text {z }}$, T. Kress ${ }^{\text {d }}$, P. Krieger ${ }^{\text {f }}$, J. von Krogh ${ }^{\text {k }}$, T. Kuhl ${ }^{\text {c }}$, M. Kupper ${ }^{x}$, P. Kyberd ${ }^{\text {m }}$, G.D. Lafferty ${ }^{\text {p }}$, H. Landsman ${ }^{\text {u, D. Lanske }}{ }^{\text {n, }}$ I. Lawson ${ }^{\mathrm{z}}$, J.G. Layter ${ }^{\mathrm{d}}$, A. Leins ${ }^{\text {ae }}$, D. Lellouch ${ }^{\mathrm{x}}$, J. Letts ${ }^{1}$, L. Levinson ${ }^{\mathrm{x}}$, R. Liebisch ${ }^{\text {k }}$, J. Lillich ${ }^{\mathrm{j}}$, B. List ${ }^{\mathrm{h}}$, C. Littlewood ${ }^{\mathrm{e}}$, A.W. Lloyd ${ }^{\mathrm{a}}$, S.L. Lloyd ${ }^{\mathrm{m}}$, F.K. Loebinger ${ }^{\mathrm{p}}$, G.D. Long ${ }^{\mathrm{z}}$, M.J. Losty ${ }^{\mathrm{g}}$, J. Lu ${ }^{\text {aa }}$, J. Ludwig ${ }^{\mathrm{j}}$, A. Macchiolo ${ }^{\mathrm{r}}$, A. Macpherson ${ }^{\text {ab }}$, W. Mader ${ }^{\text {c }}$, M. Mannelli ${ }^{\text {h }}$, S. Marcellini ${ }^{b}$, T.E. Marchant ${ }^{p}$, A.J. Martin ${ }^{\text {m }}$, J.P. Martin ${ }^{\mathrm{r}}$, G. Martinez ${ }^{\mathrm{q}}$, T. Mashimo ${ }^{\mathrm{w}}$, P. Mättig ${ }^{\mathrm{x}}$, W.J. McDonald ${ }^{\text {ab }}$, J. McKenna ${ }^{\text {aa }}$, T.J. McMahon ${ }^{\text {a }}$, R.A. McPherson ${ }^{\mathrm{z}}$, F. Meijers ${ }^{\mathrm{h}}$, P. Mendez-Lorenzo ${ }^{\text {ae }}$, F.S. Merritt ${ }^{\text {i }}$, H. Mes ${ }^{\text {g }}$, I. Meyer ${ }^{\text {e }}$, A. Michelini ${ }^{\text {b }}$, S. Mihara ${ }^{\text {w }}$, G. Mikenberg ${ }^{\text {x }}$, D.J. Miller ${ }^{\circ}$, W. Mohr ${ }^{\text {j, }}$, A. Montanari ${ }^{\text {b }}$, T. Mori ${ }^{\text {w }}$,

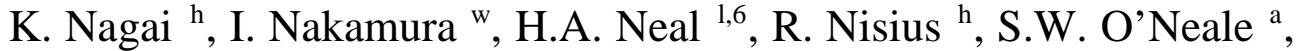
F.G. Oakham ${ }^{\text {g }}$, F. Odorici ${ }^{\text {b }}$, H.O. Ogren ${ }^{1}$, A. Okpara ${ }^{\text {k }}$, M.J. Oreglia ${ }^{\text {i }}$, S. Orito ${ }^{\text {w, }}$ G. Pásztor ${ }^{\text {ac }}$, J.R. Pater ${ }^{\mathrm{p}}$, G.N. Patrick ${ }^{\mathrm{t}}$, J. Patt ${ }^{\mathrm{j}}$, R. Perez-Ochoa ${ }^{\mathrm{h}}$, P. Pfeifenschneider ${ }^{\text {n }}$, J.E. Pilcher ${ }^{\text {i }}$, J. Pinfold ${ }^{\text {ab }}$, D.E. Plane ${ }^{\text {h }}$, B. Poli ${ }^{\text {b }}$, J. Polok ${ }^{\text {h }}$, M. Przybycień ${ }^{\text {h,7 }}$, A. Quadt ${ }^{\text {h }}$, C. Rembser ${ }^{\text {h }}$, H. Rick ${ }^{\text {h }}$, S.A. Robins ${ }^{\text {u, }}$ N. Rodning ${ }^{\mathrm{ab}}$, J.M. Roney ${ }^{\mathrm{z}}$, S. Rosati ${ }^{\mathrm{c}}$, K. Roscoe ${ }^{\mathrm{p}}$, A.M. Rossi ${ }^{\mathrm{b}}$, Y. Rozen ${ }^{\mathrm{u}}$, K. Runge ${ }^{\text {j, O. Runolfsson }}{ }^{\text {h, D.R. Rust }}{ }^{1}$, K. Sachs ${ }^{\text {j}}$, T. Saeki ${ }^{\text {w }}$, O. Sahr ${ }^{\text {ae }}$, W.M. Sang ${ }^{y}$, E.K.G. Sarkisyan ${ }^{\text {v }}$, C. Sbarra ${ }^{\text {z }}$, A.D. Schaile ${ }^{\text {ae }}$, O. Schaile ${ }^{\text {ae }}$, P. Scharff-Hansen ${ }^{\text {h }, ~ S . ~ S c h m i t t ~}{ }^{k}$, A. Schöning ${ }^{\text {h }}$, M. Schröder ${ }^{\text {h }}$, M. Schumacher ${ }^{y}$, C. Schwick ${ }^{\text {h}}$, W.G. Scott ${ }^{t}$, R. Seuster ${ }^{\text {n,3 }}$, T.G. Shears ${ }^{\text {h}}$, B.C. Shen ${ }^{\text {d }}$, C.H. Shepherd-Themistocleous ${ }^{\text {e }}$, P. Sherwood ${ }^{\circ}$, G.P. Siroli ${ }^{b}$, A. Skuja ${ }^{\text {, }}$, A.M. Smith ${ }^{\text {h }}$, G.A. Snow ${ }^{\text {q }}$, R. Sobie ${ }^{\text {z }}$, S. Söldner-Rembold j,8, S. Spagnolo ${ }^{\text {t }}$, M. Sproston ', A. Stahl c ${ }^{\text {c }}$ K. Stephens ${ }^{\text {p }}$, K. Stoll ${ }^{j}$, D. Strom ${ }^{\text {s }}$, R. Ströhmer ${ }^{\text {ae}}$, B. Surrow ${ }^{\text {h }}$, S.D. Talbot ${ }^{\text {a }}$, S. Tarem ${ }^{\text {u }}$, R.J. Taylor ${ }^{\circ}$, R. Teuscher ${ }^{\text {i }}$, M. Thiergen ${ }^{j}$, J. Thomas ${ }^{\circ}$, M.A. Thomson ${ }^{\text {h }}$, E. Torrence ${ }^{\text {h }}$, S. Towers ${ }^{\mathrm{f}}$, T. Trefzger ${ }^{\text {ae }}$, I. Trigger ${ }^{\mathrm{h}}$, Z. Trócsányi ${ }^{\text {ad,9 }}$, E. Tsur ${ }^{\mathrm{v}}$, M.F. Turner-Watson ${ }^{\text {a }}$, I. Ueda ${ }^{\mathrm{w}}$, R. Van Kooten ${ }^{1}$, P. Vannerem ${ }^{j}$, M. Verzocchi ${ }^{\text {h }}$, H. Voss ${ }^{c}$, D. Waller ${ }^{\text {f }}$, C.P. Ward ${ }^{\mathrm{e}}$, D.R. Ward ${ }^{\mathrm{e}}$, P.M. Watkins ${ }^{\mathrm{a}}$, A.T. Watson ${ }^{\mathrm{a}}$, N.K. Watson ${ }^{\mathrm{a}}$, P.S. Wells ${ }^{\text {h }}$, T. Wengler ${ }^{\text {h }}$, N. Wermes ${ }^{c}$, D. Wetterling ${ }^{k}$, J.S. White ${ }^{\text {f }}$, G.W. Wilson ${ }^{\mathrm{p}}$, J.A. Wilson ${ }^{\mathrm{a}}$, T.R. Wyatt ${ }^{\mathrm{p}}$, S. Yamashita ${ }^{\mathrm{w}}$, V. Zacek ${ }^{\mathrm{r}}$, D. Zer-Zion ${ }^{\mathrm{h}}$

${ }^{a}$ School of Physics and Astronomy, University of Birmingham, Birmingham B15 2TT, UK

${ }^{\mathrm{b}}$ Dipartimento di Fisica dell' Università di Bologna and INFN, I-40126 Bologna, Italy ${ }^{c}$ Physikalisches Institut, Universität Bonn, D-53115 Bonn, Germany

${ }^{\mathrm{d}}$ Department of Physics, University of California, Riverside, CA 92521, USA

${ }^{\mathrm{e}}$ Cavendish Laboratory, Cambridge CB3 OHE, UK

${ }^{\mathrm{f}}$ Ottawa-Carleton Institute for Physics, Department of Physics, Carleton University, Ottawa, Ont. K1S 5B6, Canada

${ }^{\mathrm{g}}$ Centre for Research in Particle Physics, Carleton University, Ottawa, Ont. K1S 5B6, Canada

${ }^{\mathrm{h}}$ CERN, European Organisation for Particle Physics, CH-1211 Geneva 23, Switzerland

${ }^{\mathrm{i}}$ Enrico Fermi Institute and Department of Physics, University of Chicago, Chicago, IL 60637, USA

${ }^{\mathrm{j}}$ Fakultät für Physik, Albert Ludwigs Universität, D-79104 Freiburg, Germany 


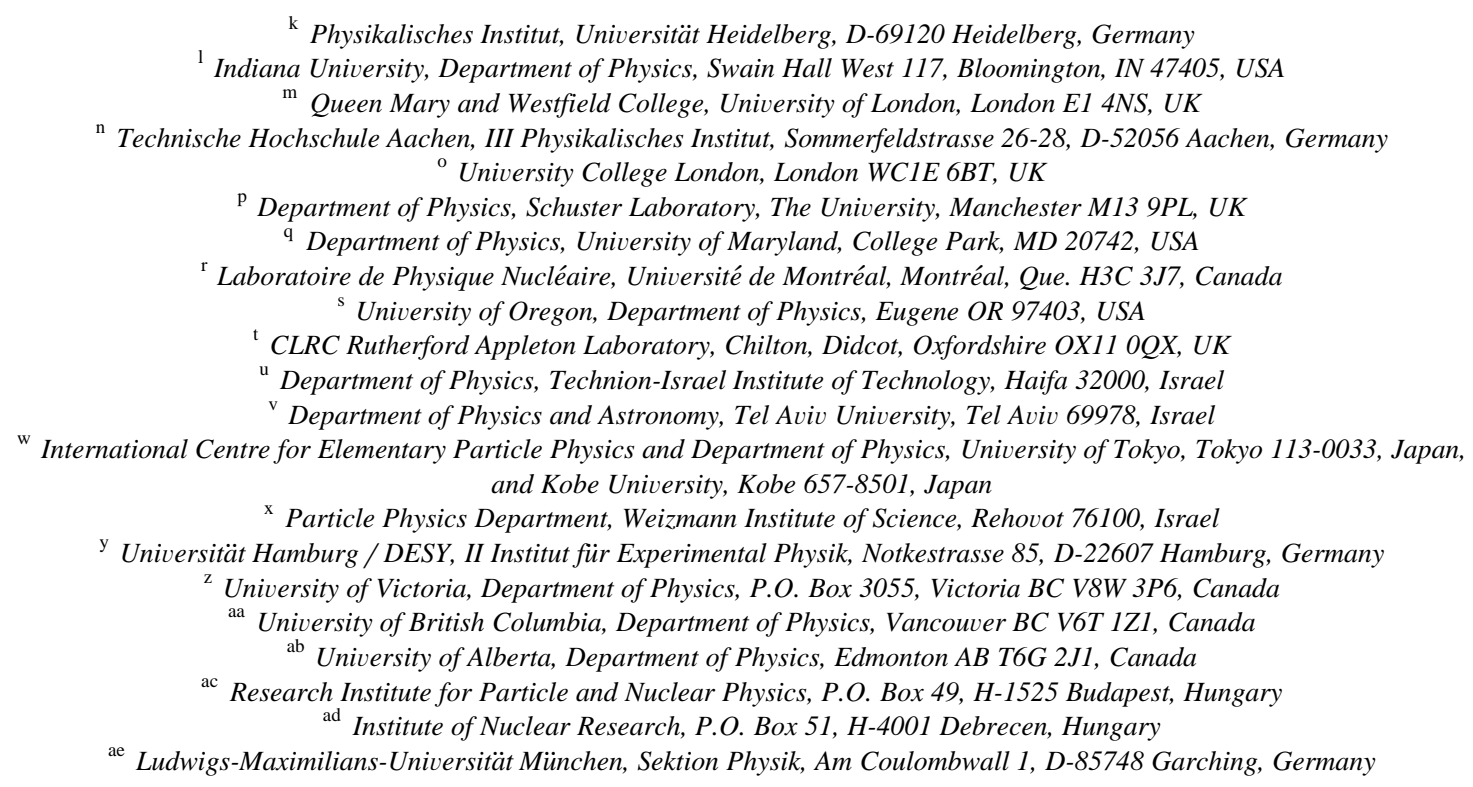

Received 17 December 1999; accepted 31 January 2000

Editor: K. Winter

\begin{abstract}
A search for the decay $\mathrm{B}^{ \pm} \rightarrow \mathrm{K}^{ \pm} \mathrm{K}^{ \pm} \pi^{\mp}$ was performed using data collected by the OPAL detector at LEP. These decays are strongly suppressed in the Standard Model but could occur with a higher branching ratio in supersymmetric models, especially in those with R-parity violating couplings. No evidence for a signal was observed and a $90 \%$ confidence level upper limit of $1.29 \times 10^{-4}$ was set for the branching ratio. (C) 2000 Published by Elsevier Science B.V. All rights reserved.
\end{abstract}

\section{Introduction}

Rare $b$ decays offer an opportunity to discover new physics beyond the Standard Model (SM). Many studies have been done in recent years to predict

\footnotetext{
${ }^{1}$ And at TRIUMF, Vancouver, Canada V6T 2A3.

${ }^{2}$ Now at MPI für Physik, 80805 München.

${ }^{3}$ And MPI München.

${ }^{4}$ And Royal Society University Research Fellow.

${ }^{5}$ And Institute of Nuclear Research, Debrecen, Hungary.

${ }^{6}$ Now at Yale University, Dept of Physics, New Haven, USA.

${ }^{7}$ And University of Mining and Metallurgy, Cracow.

${ }^{8}$ And Heisenberg Fellow.

${ }^{9}$ And Department of Experimental Physics, Lajos Kossuth University, Debrecen, Hungary.
}

flavor changing neutral current (FCNC) processes, both within the SM and beyond [1]. One of these FCNC induced processes, $\mathrm{b} \rightarrow \mathrm{s} \gamma$, has been measured [2] and the branching ratio found to be consistent with the SM prediction [3]. However, significant uncertainties still remain in both the theoretically predicted branching ratio and the measurement. Thus it is hard to conclude if this process shows signs of new physics [4]. This is also true in most of the channels such as $\mathrm{b} \rightarrow \mathrm{sq} \bar{q}$ [5] and $\mathrm{b} \rightarrow \mathrm{s} \ell \bar{\ell}$ [6], due to theoretical uncertainties.

The process $\mathrm{b} \rightarrow \mathrm{ss} \overline{\mathrm{d}}$, induced by a box diagram, is predicted to be exceedingly small in the SM (Fig. 1(a)), of the order of $10^{-11}$ [7]. However, in the minimal supersymmetric standard model (MSSM) [8], this transition can be induced by the squarkgaugino (or higgsino) box diagrams (Fig. 1(b)) at a 
(a)

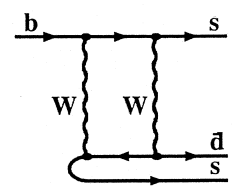

(b)

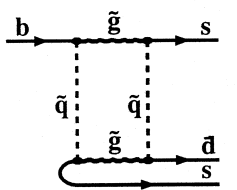

(c)

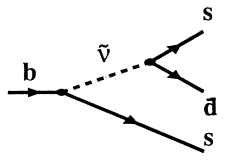

Fig. 1. $b \rightarrow$ ss $\bar{d}$ transition (a) SM, (b) MSSM, (c) MSSM with R-parity violating coupling.

level of $10^{-7}-10^{-8}$. An alternative mechanism for this channel in supersymmetric models is through R-parity violating couplings [9] (Fig. 1(c)). These two possibilities appear to be the only ones that will produce significant enhancement of this decay within supersymmetric models [7]. Two higgs doublet models could also induce this decay at branching ratios significantly larger than in the Standard Model, for a certain range of the parameters involved [10].

Typical exclusive processes of $b \rightarrow$ ss $\bar{d}$ include $\mathrm{B}^{ \pm} \rightarrow \mathrm{K}^{ \pm} \mathrm{K}^{0}$, which are difficult to separate from the standard penguin process $\mathrm{b} \rightarrow \mathrm{ds} \overline{\mathrm{s}}$. Although the interference of these two sources of the final state is crucial in the study of phenomena such as $\mathrm{CP}$ violation, this channel is not suitable for a direct search for new physics. However the decay $\mathrm{B}^{-} \rightarrow$ $\mathrm{K}^{-} \mathrm{K}^{-} \pi^{+}{ }^{10}$, either as a direct three-body decay or through a $\mathrm{K}^{\star}$-like resonance, is a clear signature of this process. This document describes the first search for the decay $\mathrm{B}^{-} \rightarrow \mathrm{K}^{-} \mathrm{K}^{-} \pi^{+}$.

\section{Hadronic event selection and simulation}

We used data collected at LEP by the OPAL detector [11] between 1990 and 1995 running at center-of-mass energies in the vicinity of the $\mathrm{Z}^{0}$ peak. Hadronic $Z^{0}$ decays were selected using the number of charged tracks and the visible energy in each event as in Ref. [12]. This selection yielded 4.41 million hadronic events.

Monte Carlo events were used to determine the selection efficiency, for training of an artificial neural network (ANN) used in selecting the final event sample (Section 3), and for the determination of

\footnotetext{
${ }^{10}$ Charge conjugation is assumed throughout this paper
}

some of the systematic uncertainties (Section 4). To determine the selection efficiency we generated 100000 Monte Carlo events of the process $Z^{0} \rightarrow b \bar{b}$ where one of the $\mathrm{b}$ quarks hadronised into $\mathrm{a}^{-}$ meson which subsequently decayed to $\mathrm{K}^{-} \mathrm{K}^{-} \pi^{+}$. One sample was generated according to three-body decay phase space, while another sample was generated with angular distribution as expected from a weak decay matrix element. In addition, we generated samples in which the B meson decayed via an intermediate $K^{\star}$ resonance. $K^{\star}(892), K^{\star}(1680)$ and $\mathrm{K}^{\star}(2045)$ resonances were chosen as they all decay into $\mathrm{K}^{-} \pi^{+}$and cover the entire spectrum of $\mathrm{K}^{\star}$ resonances.

For optimisation of the selection of events and for some of the studies of systematic uncertainties, we generated 4 million 5-flavour hadronic $\mathrm{Z}^{0}$ decays (referred to as $\mathrm{q} \overline{\mathrm{q}}$ Monte Carlo). All these samples were generated with the JETSET 7.4 Monte Carlo program [13] with parameters tuned to the OPAL data [14]. The heavy quark fragmentation was parameterised by the fragmentation function of Peterson et al. [15], and all samples were processed with the OPAL detector simulation package [16].

\section{Analysis procedure}

In each event, charged tracks and electromagnetic clusters not associated to a charged track were combined into jets, using the JADE algorithm with the E0 recombination scheme [17]. Within this algorithm jets are defined by $y_{\text {cut }}=0.04$, where $y_{\text {cut }}$ is defined in Ref. [17].

The primary vertex of the event was reconstructed using the charged tracks in the event and the knowledge of the position and spread of the $\mathrm{e}^{+} \mathrm{e}^{-}$collision point. 
Table 1

Summary of $\mathrm{d} E / \mathrm{d} x$ selection criteria: $\mathrm{d} E / \mathrm{d} x_{\pi(K)}$ is the difference between the measured value of the ionisation energy loss in the jet chamber and the expected value for $\pi(\mathrm{K})$ and $\sigma$ represents the expected standard deviation of the distribution. $N_{\mathrm{d} E / \mathrm{d} x}$ is the number of jet chamber hits used for $\mathrm{d} E / \mathrm{d} x$. The $\mathrm{d} E / \mathrm{d} x$ cuts were chosen based on probability values.

\begin{tabular}{lcc}
\hline Selection & $\pi$ selection & K selection \\
\hline $\mathrm{d} E / \mathrm{d} x_{\pi}$ & - & $<-1.29 \sigma$ \\
$\mathrm{d} E / \mathrm{d} x_{K}$ & $>1.15 \sigma$ & $\pm 1.55 \sigma$ \\
$\mathrm{d} E / \mathrm{d} x_{p}$ & - & $>0$ \\
$N_{\mathrm{d} E / \mathrm{d} x}$ & $>20$ & $>20$ \\
\hline
\end{tabular}

We searched the hadronic event sample for the decay $\mathrm{B}^{-} \rightarrow \mathrm{K}^{-} \mathrm{K}^{-} \pi^{+}$by combining three charged tracks to form a $\mathrm{B}$ meson candidate. All three track combinations were considered. All tracks were required to have a momentum of at least $2 \mathrm{GeV} / c$ and to be in the same jet. Two of the tracks were required to have the same charge and were assigned the mass of a kaon. A third track, with an opposite charge, was assumed to be the pion. Tracks were required to satisfy selection criteria based on the measured rate of energy loss due to ionisation $(\mathrm{d} E / \mathrm{d} x)$ [18] as listed in Table 1. These $\mathrm{d} E / \mathrm{d} x$ selection criteria are $44 \%$ efficient while rejecting $98.5 \%$ of the background.

The three tracks were fitted to a common vertex and the decay length, the distance from the $\mathrm{e}^{+} \mathrm{e}^{-}$ interaction point to the reconstructed secondary vertex, was calculated. Candidates where the secondary vertex is in the hemisphere opposite to the candidate's jet were rejected. This criterion left $55 \%$ of the remaining background events, but kept $96 \%$ of the signal events.

Since the hadronic data sample consisted mostly of non- $b \bar{b}$ events, we suppressed these events by means of a b-tagging algorithm, based on reconstructed displaced secondary vertices. An artificial neural network with inputs based on decay length significance, vertex multiplicity and invariant mass information [19] was used to select vertices with a high probability of coming from $b$ hadron decays. Events were accepted if any of the jets were tagged by the neural network. The b-tagging selection was found to be $79 \%$ efficient, while rejecting $80 \%$ of the remaining background.
The final selection was based on an artificial neural network designed to select $\mathrm{B}^{-} \rightarrow \mathrm{K}^{-} \mathrm{K}^{-} \pi^{+}$ events while rejecting background events. We used the JETNET 3.4 program [20] with a feed-forward type net, trained with the back-propagation algorithm. The neural network used seven input parameters: the momenta of the three tracks $\left(p_{\pi}, p_{\mathrm{K}}\right)$; the $\mathrm{B}$ candidate momentum $\left(p_{\mathrm{B}}\right)$; the ratio of $\mathrm{B}$ candidate energy to the jet energy $\left(X_{\text {jet }}\right)$; the decay length; and the vertex probability, the probability of the three tracks to originate from a common vertex which is calculated using the track parameters. The neural network retains $74 \%$ of the signal events and rejects $97 \%$ of background events when selecting candidates with an ANN output above 0.9. Fig. 2 shows the distributions of the artificial neural network input parameters and the ANN output for candidates passing the above criteria. While the data and the $q \bar{q}$ Monte Carlo show good agreement, one can clearly observe the differences between these distributions and those of the signal Monte Carlo.
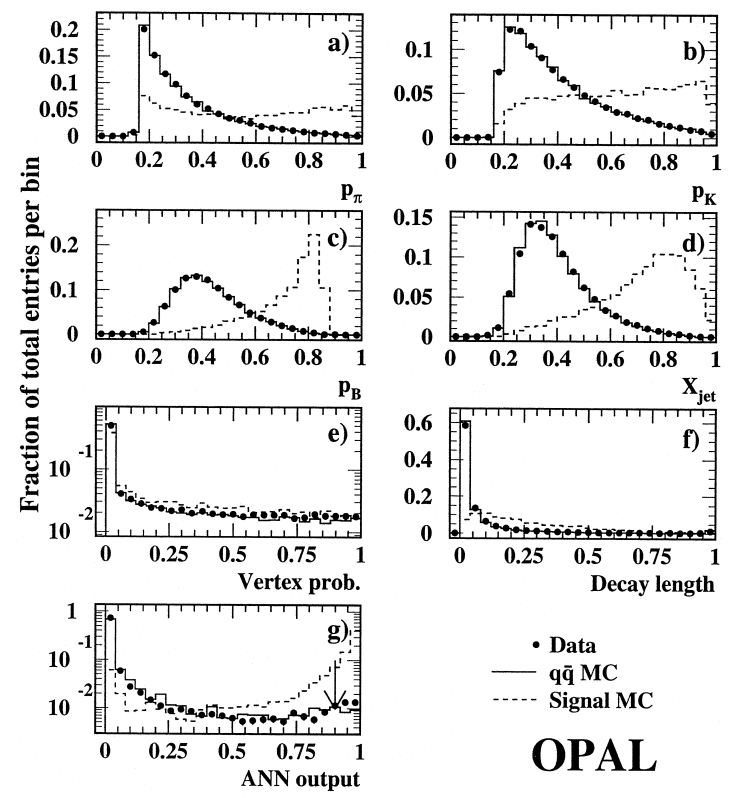

Fig. 2. Input variables to the artificial neural network (a)-(f) and output $(\mathrm{g})$. The solid line represents the $\mathrm{q} \overline{\mathrm{q}}$ Monte Carlo while the dots represent the data. The dashed line shows the distribution of $\mathrm{B}^{-} \rightarrow \mathrm{K}^{\star 0}(892) \mathrm{K}^{-}$Monte Carlo events. The arrow in (g) shows the cut value. All variables are normalised and are plotted after appropriate transformation to the range [0-1]. 
Candidates were accepted if their invariant mass was in the region: $5.10 \mathrm{GeV} / c^{2}<M_{\mathrm{KK} \pi}<5.46$ $\mathrm{GeV} / c^{2}$, which corresponds to twice the mass resolution around the nominal $\mathrm{B}^{-}$mass. Only one candidate per jet was accepted, based on the largest neural network output for candidates in a jet. Fig. 3(a) shows the invariant mass distribution of the $\mathrm{K}^{-} \mathrm{K}^{-} \pi^{+}$candidates. No enhancement is seen in the signal region, where 17 events were observed. Monte Carlo studies indicated that $88 \%$ of the background at this stage consists of $\bar{b} \bar{b}$ events.

If the decay chain $\mathrm{B}^{-} \rightarrow \mathrm{K}^{-} \mathrm{K}^{-} \pi^{+}$is assumed to be direct (i.e., without an intermediate $\mathrm{K}^{\star}$ resonance), then the mass of the $\mathrm{K}^{-} \pi^{+}$system can be exploited to further reduce background where the pion and one of the kaons are from the decay of a $\mathrm{K}^{\star}$ resonance. The mass of the $\mathrm{K}^{-} \pi^{+}$system was added as an input to the neural network, and the training procedure of the ANN was repeated. Fig. 3(b) shows the invariant mass distribution of the
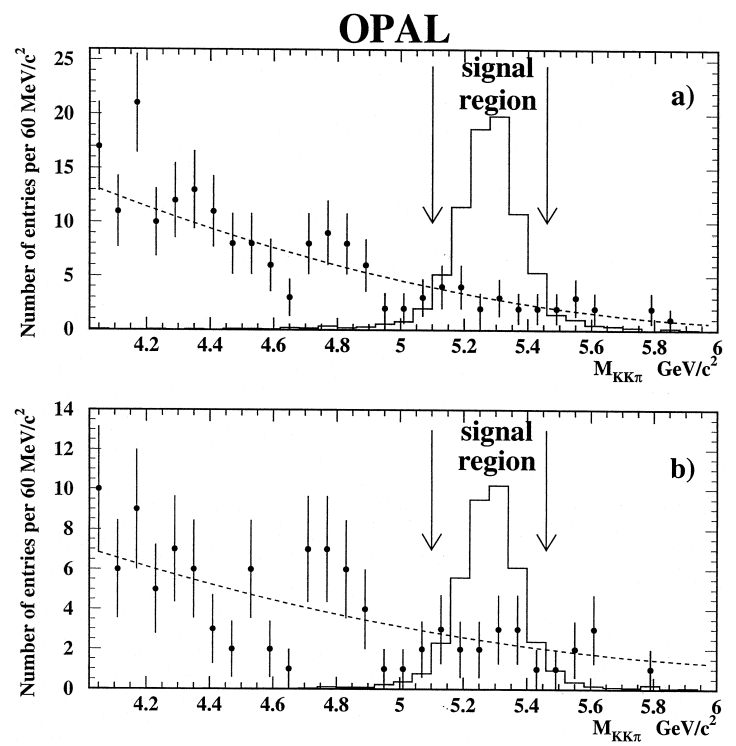

Fig. 3. Invariant mass distribution of the $\mathrm{K}^{-} \mathrm{K}^{-} \pi^{+}$candidates after all selection criteria were applied (a) via intermediate $K^{\star}$ resonance and (b) with direct production. The dots represent the data, the solid line shows the expected signal shape from Monte Carlo events after all the selection criteria were applied with arbitrary normalisation, and the dashed line is the expected background.
$\mathrm{K}^{-} \mathrm{K}^{-} \pi^{+}$candidates passing the selection. Here too, no enhancement is seen in the signal region and the observed 14 events are used to determine an upper limit on the branching ratio.

\subsection{Background estimation}

The background to the process $\mathrm{B}^{-} \rightarrow \mathrm{K}^{-} \mathrm{K}^{-} \pi^{+}$ was estimated by fitting a second-order polynomial to the invariant mass of a combinatorial background, obtained by releasing the ANN cut, and then normalising the shape to the mass side-bands of Fig. 3 (4-5 $\mathrm{GeV} / c^{2}$ and 5.6-6 $\left.\mathrm{GeV} / c^{2}\right)$. Monte Carlo studies indicated that the background shape is not altered by this procedure. Alternatively, we repeated this procedure by releasing each of the selection criteria separately and by obtaining the shape from Monte Carlo. All the alternative fits gave a consistent result. We also took the number of events within the signal region in each of the above cases and scaled it to the appropriate sample size. Here too, all estimates were consistent.

As we are setting upper limits, the conservative approach is to estimate the number of signal events using the lowest background estimate. While the fitted background shown in Fig. 3(a) gave 18.8 events in the signal region, the lowest estimate was 17.5 events. The respective numbers for the direct production case were 14.8 and 14.1 events.

\subsection{Limit determination}

The above numbers were used to determine $N^{90}$, the $90 \%$ C.L. upper limit on the number of signal events. Using the formalism of Ref. [21], we obtained $N^{90}=7.8$ and $N^{90}=7.4$ events for the resonant/direct decay, respectively.

To calculate an upper limit on the branching ratio we used:

$\mathrm{Br}\left(\mathrm{B}^{-} \rightarrow \mathrm{K}^{-} \mathrm{K}^{-} \pi^{+}\right) \leq \frac{N^{90}}{\epsilon N_{\mathrm{B}}}$,

where $N_{\mathrm{B}}$ is the number of charged B mesons in the sample and $\epsilon$ is the efficiency for Monte Carlo simulated events of the process $\mathrm{B}^{-} \rightarrow \mathrm{K}^{-} \mathrm{K}^{-} \pi^{+}$to 
survive the selection procedure. With 4.41 million hadronic $\mathrm{Z}^{0}$ decays, using $\mathrm{Br}\left(\mathrm{b} \rightarrow \mathrm{B}^{ \pm}\right)=$ $0.397_{-0.022}^{+0.018}$ and $\Gamma_{\mathrm{b} \overline{\mathrm{b}}} / \Gamma_{\text {had }}=0.2170 \pm 0.0009$ [22], we obtained $N_{\mathrm{B}}=759800_{-42200}^{+34600}$.

The conservative approach when setting upper limits is to use the model giving the lowest efficiency for the signal. If one assumes resonance production, then the lowest efficiency, $8.11 \pm 0.19 \%$, is obtained when assuming the signal decay channel is via $K^{\star 0}(892)$. The lowest efficiency for non-resonant decay, obtained with a phase space particle distribution, was found to be $11.3 \pm 0.2 \%$.

\section{Systematic uncertainties}

Systematic uncertainties may arise from the limited accuracy with which $N_{\mathrm{B}}$ is known, from the uncertainty in the simulation used to determine the efficiency and from the background estimation.

\subsection{Modelling of $d E / d x$}

To estimate the uncertainty arising from the modelling of the $\mathrm{d} E / \mathrm{d} x$ selection criteria, we compared the efficiency of the $\mathrm{d} E / \mathrm{d} x$ cuts in Monte Carlo simulated events and in data. We took advantage of the abundance and relative ease of reconstruction of $\mathrm{D}^{\star+}$ mesons, and exploited them for testing the systematic uncertainties associated with the $\mathrm{d} E / \mathrm{d} x$ selection criteria. We searched for $\mathrm{D}^{\star+}$ mesons via their decay into a $\mathrm{D}^{0}$ and a $\pi^{+}$, where the $\mathrm{D}^{0}$ decays via a $\mathrm{K}^{-} \pi^{+}$. To enhance the signal to background ratio we required the momentum of the $\mathrm{D}^{\star+}$ candidate to be larger than $15 \mathrm{GeV} / c$; the $\mathrm{D}^{\star+}$ decay vertex to be at least $50 \mu \mathrm{m}$ away from the interaction point; and the helicity angle, $\theta^{*}$, between the kaon momentum in the $\mathrm{D}$ rest frame and the $\mathrm{D}$ direction in the laboratory frame to satisfy $\cos \theta^{*}<$ 0.7. Background estimation, after applying these selection criteria, was done as in [23]. To avoid possible uncertainty due to the difference in the momentum spectrum of the $\mathrm{D}^{\star+}$ products with respect to the momentum spectrum of tracks from the process $\mathrm{B}^{-} \rightarrow \mathrm{K}^{-} \mathrm{K}^{-} \pi^{+}$, we reweighted the $\mathrm{d} E / \mathrm{d} x$ selection efficiency as a function of the track momenta. By applying the $\mathrm{d} E / \mathrm{d} x$ criteria used to select the kaon we obtained an efficiency of $56.2 \%$ in data and
$57.0 \%$ in Monte Carlo. The respective values for the pion selection efficiency are $75.0 \%$ and $76.8 \%$. Combining all numbers, the relative uncertainty on the signal efficiency associated with the $\mathrm{d} E / \mathrm{d} x$ cuts is estimated at $3.7 \%$.

\subsection{Artificial neural network uncertainty}

The agreement is good between the data and the $\mathrm{q} \overline{\mathrm{q}}$ Monte Carlo, in all of the input variables to the ANN (Fig. 2). However, the Monte Carlo simulation compared in that figure represents the background and is not used in setting the upper limit. The simulation used to set the limit is that of signal events and thus, signal input variables should be compared. This is not possible for the decay $\mathrm{B}^{-} \rightarrow$ $\mathrm{K}^{-} \mathrm{K}^{-} \pi^{+}$. Therefore, once again we made use of the $\mathrm{D}^{\star+}$ signal. We compared the kaon and pion momentum distribution, the $\mathrm{D}^{\star+}$ momentum and fraction of energy from the jet's energy, the decay length and the vertex probability. Good agreement was achieved between the data and Monte Carlo as shown in Fig. 4. Events were reweighted as a function of
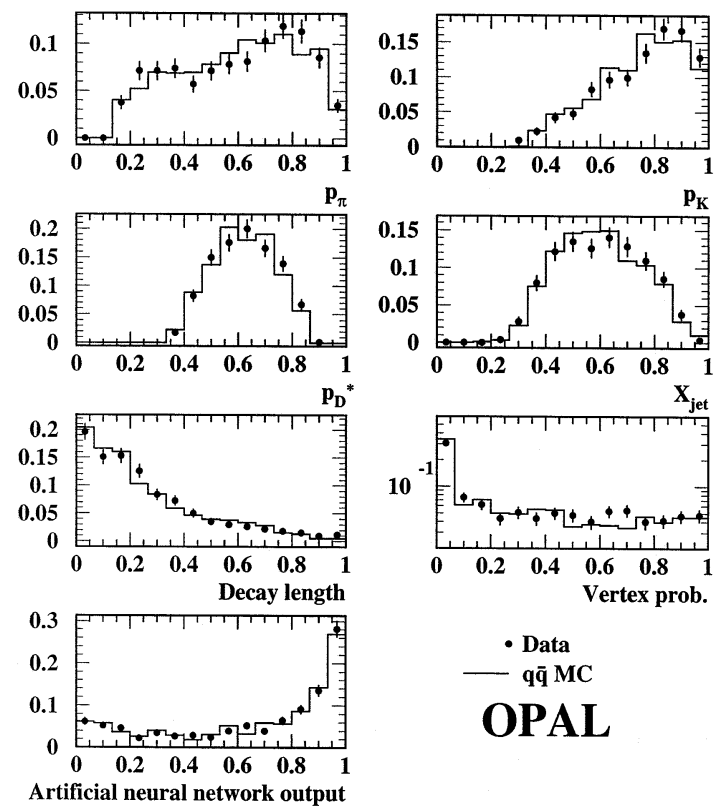

Fig. 4. Comparison of ANN input variables for $\mathrm{D}^{\star+}$ candidates. The solid line represents the $\mathrm{q} \overline{\mathrm{q}}$ Monte Carlo and the dots are the data. 
the track momenta to reflect the signal spectrum as in the $\mathrm{d} E / \mathrm{d} x$ uncertainty section, and the ANN output was evaluated. In order to assign a systematic uncertainty to the efficiency of the ANN, we took the difference between the fraction of $\mathrm{D}^{\star+}$ events passing the ANN cut in the data to that in the $q \bar{q}$ Monte Carlo. We obtained an uncertainty of $4.1 \%$. In addition, we took the difference in the ANN efficiency obtained for the signal involving different $\mathrm{K}^{\star}$ resonances. This difference was found to be $2.1 \%$ and the overall uncertainty assigned for this source was $4.6 \%$

\subsection{B hadron lifetime and decay multiplicity}

The probability to reconstruct the signal $\mathrm{B}^{-}$meson from also depends on the efficiency to reconstruct secondary vertices in both hemispheres. This in turn is sensitive to the charged decay multiplicity and lifetime of the B hadrons. The Monte Carlo was reweighted to reflect the measured multiplicities and lifetimes [22]. The uncertainty on these figures gave an uncertainty of $1.1 \%$ and $1.3 \%$, respectively, on the selection efficiency.

\subsection{Detector modelling}

The resolution of the tracking devices has an effect on the efficiency. The simulated resolutions were varied by $\pm 10 \%$ relative to the values that optimally describe the data following the studies in [24]. The analysis was repeated and the efficiency estimation was recalculated. This source contributed an uncertainty of $1.2 \%$.

\subsection{Fragmentation modelling}

The heavy-quark fragmentation was simulated using the function of Peterson et al. [15]. The heavyquark fragmentation model parameter was varied to change the mean scaled energy of weakly-decaying bottom hadrons within the experimental range: $\left\langle x_{E}\right\rangle_{\mathrm{b}}=0.702 \pm 0.008$ [22]. This change resulted in a $2 \%$ change in the efficiency. In addition, the heavy-quark fragmentation model was changed to that suggested by Collins and Spiller [25] and to that of Kartvelishvili et al. [26], with parameters tuned according to Ref. [27]. No significant change in the resulting efficiency was observed.

\subsection{Background estimation uncertainty}

The uncertainty on the fitted shape parameters and on the normalisation gave an uncertainty on the background estimate. The different techniques used to estimate the background resulted in consistent estimates with a small standard deviation (about 1 event). However, since we used the lowest background estimate, these uncertainties were not taken into account as they were smaller than the difference between the mean background estimate and the one used.

\section{Results}

Combining all sources of systematic uncertainties mentioned above, as well as the statistical uncertainty in determining the efficiency and the uncertainty on $N_{\mathrm{B}^{-}}$, we obtained an uncertainty of $8.4 \%$ on the denominator of Eq. (1). This uncertainty was incorporated according to the method outlined in Ref. [28]. With $N_{\text {res. }}^{90}=7.8$ events and $N_{\text {no res. }}^{90}=7.4$ events we obtained:

$$
\begin{aligned}
& \operatorname{Br}\left(\mathrm{B}^{-} \rightarrow \mathrm{K}^{-} \mathrm{K}^{-} \pi^{+}\right) \quad \leq 1.29 \times 10^{-4} @ 90 \% \text { C.L. } \\
& \operatorname{Br}\left(\mathrm{B}^{-} \rightarrow \mathrm{K}^{-} \mathrm{K}^{-} \pi^{+}\right) \text {non-resonance } \leq 8.79 \times 10^{-5} @ 90 \% \mathrm{C} . \mathrm{L} .
\end{aligned}
$$

\section{Summary}

We have searched for the decay of charged $\mathrm{B}$ mesons to $\mathrm{K}^{-} \mathrm{K}^{-} \pi^{+}$. This decay channel is strongly suppressed in the Standard Model, but may be large in R-parity violating models. Hence, this decay mode may serve as a probe for new physics beyond the Standard Model. No evidence has been observed for such a decay. Upper limits on the branching ratio have been set of $1.29 \times 10^{-4}$, or of $8.79 \times 10^{-5}$ if one assumes that the decay is not via a $\mathrm{K}^{\star}$ resonance, both at $90 \%$ confidence level.

Using these limits, and the estimate $\frac{\mathrm{B}^{-} \rightarrow \mathrm{K}^{-} \mathrm{K}^{-} \pi^{+}}{\mathrm{b} \rightarrow \mathrm{ss} \overline{\mathrm{d}}} \approx$ $\frac{1}{4}$ [7], we can put new limits on the contribution of 
R-parity violating couplings in this process. Starting from Eq. (9) of Ref. [7],

$$
\begin{aligned}
\Gamma= & \frac{m_{\mathrm{b}}^{5} f_{\mathrm{QCD}}^{2}}{512(2 \pi)^{3}}\left(\left|\sum_{\mathrm{n}=1}^{3} \frac{1}{m_{\tilde{\nu}_{\mathrm{n}}}^{2}} \lambda_{\mathrm{n} 32}^{\prime} \lambda_{\mathrm{n} 21}^{\star \star}\right|^{2}\right. \\
& \left.+\left|\sum_{\mathrm{n}=1}^{3} \frac{1}{m_{\tilde{\nu}_{\mathrm{n}}}^{2}} \lambda_{\mathrm{n} 12}^{\prime} \lambda_{\mathrm{n} 23}^{\prime \star}\right|^{2}\right),
\end{aligned}
$$

where $m_{\mathrm{b}}$ is the mass of the $\mathrm{b}$ quark, $f_{\mathrm{QCD}}=$ $\left(\alpha_{s}\left(m_{\mathrm{b}}\right) / \alpha_{s}\left(m_{\tilde{\nu}_{\mathrm{n}}}\right)\right)^{24 / 23}, m_{\tilde{\nu}_{\mathrm{n}}}$ is the mass of the sneutrino involved and $\lambda^{\prime}$ is a dimensionless coupling. As an example, with $m_{\mathrm{b}}=4.5 \mathrm{GeV} / c^{2}, f_{\mathrm{QCD}} \simeq 2$, $m_{\tilde{\nu}_{\mathrm{n}}}=100 \mathrm{GeV} / c^{2}$ as in [7] and $\tau_{\mathrm{B}^{-}}=1.65 \mathrm{ps}$ we obtain:

$$
\sqrt{\left|\sum_{\mathrm{n}=1}^{3} \lambda_{\mathrm{n} 32}^{\prime} \lambda_{\mathrm{n} 21}^{\prime \star}\right|^{2}+\left|\sum_{\mathrm{n}=1}^{3} \lambda_{\mathrm{n} 12}^{\prime} \lambda_{\mathrm{n} 23}^{\prime \star}\right|^{2}}<5.9 \times 10^{-4},
$$

which can be compared to the existing limit of 0.1 obtained from $\mathrm{b} \rightarrow$ ss $\overline{\mathrm{d}}$ [7] and neutrino mass calculation [29].

\section{Acknowledgements}

The authors would like to acknowledge Prof. Paul Singer for valuable discussion related to the analysis presented here. We particularly wish to thank the SL Division for the efficient operation of the LEP accelerator at all energies and for their continuing close cooperation with our experimental group. We thank our colleagues from CEA, DAPNIA/SPP, CE-Saclay for their efforts over the years on the time-of-flight and trigger systems which we continue to use. In addition to the support staff at our own institutions we are pleased to acknowledge the

- Department of Energy, USA,

- National Science Foundation, USA,

- Particle Physics and Astronomy Research Council, UK,

- Natural Sciences and Engineering Research Council, Canada,

- Israel Science Foundation, administered by the Israel Academy of Science and Humanities,

- Minerva Gesellschaft,

- Benoziyo Center for High Energy Physics,

- Japanese Ministry of Education, Science and Culture (the Monbusho) and a grant under the Monbusho International Science Research Program,
- Japanese Society for the Promotion of Science (JSPS),

- German Israeli Bi-national Science Foundation (GIF),

- Bundesministerium für Bildung, Wissenschaft, Forschung und Technologie, Germany,

- National Research Council of Canada,

- Research Corporation, USA,

- Hungarian Foundation for Scientific Research, OTKA T-029328, T023793 and OTKA F-023259.

\section{References}

[1] For reviews, see A. Masiero, L. Silvestrini, SUSY in rare and CP-violating B decays, hep-ph/9709244; J.L. Hewett, Probing supersymmetry in rare B decays, in: Proc. 28th International Conference on High-energy Physics, Warsaw, Poland, 25-31 July 1996, ICHEP 96, p. 1143.

[2] CLEO Collaboration, R. Ammar et al., Phys. Rev. Lett. 71 (1993) 674; CLEO Collaboration, M.S. Alam et al., Phys. Rev. Lett. 74 (1995) 2885; ALEPH Collaboration, R. Barate et al., Phys. Lett. B 429 (1998) 169.

[3] K. Chetyrkin, M. Misiak, M. Munz, Phys. Lett. B 400 (1997) 206; A.J. Buras, A. Kwiatkowski, N. Pott, Phys. Lett. B 414 (1997) 157.

[4] J.L. Hewett, Phys. Rev. Lett. 70 (1993) 1045.

[5] L.L. Chau, H.Y. Cheng, W.K. Sze, H. Yao, B. Tseng, Phys. Rev. D 43 (1991) 2176; C.-D. Lü, D.-X. Zhang, Phys. Lett. B 400 (1997) 188.

[6] C.-D. Lü, D.-X. Zhang, Phys. Lett. B 397 (1997) 279; G. Buchalla, G. Isidori, Nucl. Phys. B 525 (1998) 333; see also P. Singer, in: M. Beyer, T. Mannel, H. Schroeder (Eds.), Proc. 4th International Workshop on Progress in Heavy Quark Physics, Rostock, Germany, Sep 1997, p. 225.

[7] K. Huitu, D.-X. Zhang, C.-D. Lü, P. Singer, Phys. Rev. Lett. 81 (1998) 4313.

[8] H.P. Nilles, Phys. Rep. 110 (1984) 1; H.E. Haber, G.L. Kane, Phys. Rep. 117 (1985) 75.

[9] P. Fayet, in: S. Ferrara, J. Ellis, P. Van Nieuewenhuizen (Eds.), Unification of the Fundamental Particle Interactions, Plenum Press, 1980, p. 727.

[10] K. Huitu, C.-D. Lü, P. Singer, D.-X. Zhang, Phys. Lett. B 445 (1999) 394.

[11] OPAL Collab., K. Ahmet et al., Nucl. Instr. Meth. A 305 (1991) 275; OPAL Collab., P.P. Allport et al., Nucl. Instr. Meth. A 324 (1993) 34; OPAL Collab., P.P. Allport et al., Nucl. Instr. Meth. A 346 (1994) 476.

[12] OPAL Collab., G. Alexander et al., Z. Phys. C 52 (1991) 175.

[13] T. Sjöstrand, Comp. Phys. Comm. 82 (1994) 74; T. Sjöstrand, Comp. Phys. Comm. 39 (1986) 347; M. Bengtsson, T. Sjöstrand, Comp. Phys. Comm. 43 (1987) 367; T. Sjöstrand, Int. J. of Mod. Phys. A 3 (1988) 751. 
[14] OPAL Collab., G. Alexander et al., Z. Phys. C 69 (1996) 543.

[15] C. Peterson, D. Schlatter, I. Schmitt, P. Zerwas, Phys. Rev. D 27 (1983) 105.

[16] J. Allison et al., Nucl. Instr. Meth. A 317 (1992) 47.

[17] JADE Collaboration, W. Bartel et al., Z. Phys. C 33 (1986) 23; JADE Collaboration, S. Bethke et al., Phys. Lett. B 213 (1988) 235.

[18] M. Hauschild et al., Nucl. Instr. Meth. A 314 (1992) 74.

[19] OPAL Collaboration, R. Akers et al., Z. Phys. C 66 (1995) 19.

[20] L. Lonnblad, C. Peterson, T. Rognvaldsson, Comp. Phys. Comm. 70 (1992) 167.

[21] G.J. Feldman, R.D. Cousins, Phys. Rev. D 57 (1998) 3873.

[22] The LEP Collaborations, ALEPH, DELPHI, L3 and OPAL, Nucl. Instr. and Meth. A 378 (1996) 101; updated averages are given in 'Input Parameters for the LEP Electroweak Heavy Flavour Results for Summer 1998 Conferences', LEPHF 98-01 (see http://www.cern.ch/LEPEWWG/ heavy/); A Combination of Preliminary Electroweak Mea- surements and Constraints on the Standard Model, ALEPH, DELPHI, L3 and OPAL Collaborations, the LEP Electroweak Working Group and the SLD Heavy Flavour and Electroweak Groups, CERN-EP/99-015.

[23] 'Measurement of the Production Rate of Charm Quark Pairs from Gluons in Hadronic $\mathrm{Z}^{0}$ Decays', OPAL Collab., G. Abbiendi et al., CERN-EP/99-089, accepted for publication in Eur. Phys. J.

[24] OPAL Collaboration, G. Abbiendi et al., Eur. Phys. J. C 8 (1999) 217.

[25] P. Collins, T. Spiller, J. Phys. G 11 (1985) 1289.

[26] V.G. Kartvelishvili, A.K. Likehoded, V.A. Petrov, Phys. Lett. B 78 (1978) 615.

[27] OPAL Collaboration, G. Alexander et al., Phys. Lett. B 364 (1995) 93; OPAL Collaboration, G. Alexander et al., Z. Phys. C 72 (1996) 1.

[28] R.D. Cousins, V.L. Highland, Nucl. Instr. Meth. A 320 (1992) 331.

[29] G. Bhattacharyya, P.B. Pal, Phys. Rev. D 59 (1999) 091701. 